\title{
Interaction between Technology and Extraction Scaling Real Options in Natural Gas Production
}

\author{
Paul Enders, Alan Scheller-Wolf, Nicola Secomandi \\ Tepper School of Business, Carnegie Mellon University, \\ 5000 Forbes Avenue, Pittsburgh, Pennsylvania 15213, United States, \\ \{penders,awolf,ns7\}@andrew.cmu.edu \\ Tepper Working Paper 2008-E04 \\ Submitted: April 2009; Revised: January 2010
}

\begin{abstract}
This paper is the outcome of a research engagement studying questions of technology utilization and production management with managers at EQT Corp., an integrated natural gas production and distribution company. The question of how to best leverage the use of technology is fundamental to almost any industry; this is especially true for those companies operating in the volatile field of commodities production, as EQT Corp. does. We consider the interaction between two types of real options that arise in natural gas production: The option to scale the production level, through enhanced extraction and communication technologies, and the option to scale the extraction rate, by pausing production. We study this interaction by applying stochastic dynamic programming to actual operational and financial data. Our analysis brings to light data-driven managerial principles pertaining to the valuation and deployment of these three scaling options, the effect of price uncertainty on the option values, how to effectively simplify the optimal deployment policy, and whether these options, or subsets of them, are complements or substitutes. These principles are significant to natural gas production managers and, potentially, to managers of other natural resource production processes, such as the extraction of oil and mining.
\end{abstract}

\section{Introduction}

One of the fundamental questions in almost any industry is how to best leverage the use of technology; companies are typically faced with an assortment of technology options and must decide how to dynamically manage them to maximize the value of their assets. This is particularly true in the extraction industry: For example production managers at an oil or natural gas production company are constantly challenged with evaluating technology adoption choices to deal with liquid load-up, a phenomenon that retards extraction from a well.

Two types of technologies that help deal with liquid load-up in natural gas wells are (i) flow enhancement technology (ET) that increases the instantaneous production level as well as the total extractable reserves, and (ii) communication technology (CT) that increases operational efficiency, 
boosting the instantaneous production level, but also increasing the rate at which a reservoir is depleted. ET examples include continuous flow, whereby one simply uses the gas flow to bring liquids to the surface, and soap and blow, which comprises periodically manually dropping a soapstick into the well to transform the liquids into foam, which is more easily carried to the surface by the gas flow. CT examples include manually collecting production (flow rate) data and monitoring a well's status (operating or not) on a periodic basis by a human operator, and automated continuous data collection and monitoring of the well through electronic data transmission.

These examples illustrate that ET and CT can be deployed at different "levels" that can be modified over time. These modifications may be contingent on the natural gas flow rate and the prevailing natural gas price, which fluctuates over time, as well as the technology adjustment costs, which may be seasonal. Thus, one can interpret the choices of ET and CT levels as real options to scale the production level (Trigeorgis 1999, p. 2). A third real option that is available to natural gas production managers, and more generally commodity production managers, is the option to scale the extraction rate by pausing production. This option consists of temporarily ceasing production from a well, often in anticipation of an increase in price.

While versions of these three real options have been considered in separate streams of literature, the study of their interaction has received very little attention. We explore the nature of this interaction in cooperation with production managers at EQT Corp., an integrated energy company with emphasis on natural gas supply, transmission, and distribution in the Appalachian area (www.eqt.com). To do so we develop and analyze a stochastic dynamic programming model, with uncertain natural gas price evolution modeled as in Jaillet et al. (2004). Our work significantly extends the existing models of well behavior (e.g., Tarek 2006) to capture how modifying technology levels affects the flow rate evolution. Our work also stands out for its unique use of a novel data set that includes over 20 years of production data from a natural gas well that is part of the Eastern Kentucky production portfolio of EQT Corp. Our model integrates this production data with financial data describing the well's cost structure and natural gas price data available from the New York Mercantile Exchange (NYMEX), in order to establish the marginal and joint values of the three scaling options. Our model also includes the option to abandon a well, but we do not focus on its valuation, as our main interest is on how these three scaling options interact with each other. 
We find that all options, especially the ET scaling option, have substantial value by themselves. That the application of technology to natural gas extraction could add significant value is to be expected. However, our finding that pausing could have significant value, under certain conditions, was surprising to the EQT Corp. managers who participated in this study. More broadly, pausing is often ignored in practice (Slade 2001, Dugan 2006, Sciullo 2006, Al-Harthy 2007), because the conventional wisdom among managers is often to maximize the production rate, under the premise that the value of any gas not produced during a given time period is effectively lost due to financial discounting.

When used together, the ET and CT scaling options behave as complements, in the sense that each option is worth more when used in combination with the other option than when used alone. This is also true of the combination of CT scaling and pausing options. In contrast, the ET scaling and pausing options behave as substitutes. Moreover, the ET scaling option and the portfolio that includes the CT scaling and pausing options are complements, and so are the CT scaling option and the portfolio that includes the ET scaling and pausing options; this means that each technology scaling option is worth more when used together with the remaining technology and extraction scaling options rather than alone. In contrast, the pausing option and the portfolio that includes the ET and CT scaling options are substitutes. Finally, the option value of the portfolio that includes all the technology and extraction scaling options exceeds the sum of the individual values of these options. The insights behind these results are not immediate; we provide detailed supportive explanations.

We also find that stochastic variability in the natural gas price increases the value of all the scaling options, but in unique ways. The pausing option derives almost its entire value from this stochastic variability, which is surprising given the marked seasonality (deterministic variability) in the natural gas forward curve. In contrast, stochastic variability only slightly increases the values of the technology scaling options, which seems expected.

Finally, we show that the optimal scaling option deployment policy is rather complex. We thus seek a simpler yet effective approach to managing the portfolio of scaling options. We find that immediately setting the two technology scaling options at their highest levels and afterwards managing the pausing option in conjunction with the abandonment option yields essentially the same value as the complex optimal policy. This approach is useful because it drastically simplifies the deployment of the portfolio of scaling options with almost no decrease in value. 
Our results contribute to the practice-based literature in operations management by providing data-driven managerial principles (Fisher 2007, p. 374) pertaining to the value and management of technology and extraction scaling options in natural gas production. These principles are specifically significant to natural gas production managers and, potentially, to managers of other natural resource production processes, such as the extraction of oil and mining, two activities that encompass similar volatile environments and production processes.

We proceed as follows. We review the relevant literature in $\S 2$, introduce our model in $\S 3$, and discuss the data used in our study in $\S 4$. We present our results on the valuation and deployment of the technology and extraction scaling options in $\S 5$ and $\S 6$. We conclude in $\S 7$ where we also discuss limitations of our work and potential additional research avenues.

\section{Literature Review}

Early on, Grayson (1960) recognized the need for a quantitative approach to support drilling decisions by oil and gas operators, to step away from rules of thumb and pure expert judgment that were commonly used in practice. Durrer and Slater (1977) provide a thorough review of operations research methods used in petroleum and natural gas production up to the late 1970's. Most of the more recent literature on the optimal operation and valuation of commodity projects, including oil and gas ventures, uses the real option approach to value and optimize managerial flexibility (Dixit and Pindyck 1994, Smith and McCardle 1999, Trigeorgis 1999).

Brennan and Schwartz (1985) consider the decision to open, close or abandon a mine using replicating portfolios. Olson and Stensland (1988) analyze the optimal shutdown decision when the extraction cost is fixed, as in Clarke and Reed (1990), who consider oil well valuation and abandonment decisions using stopping rules. Smith and McCardle (1998) use the integrated option pricing and decision analytic approach of Smith and Nau (1995) to value oil properties. They consider the decision to open, close or abandon a property, and provide thresholds for each decision in terms of production rates and prices. Lund (2000) analyzes the value of flexibility in offshore petroleum projects. Kamrad and Ernst (2001) study the optimal production policy with yield and price uncertainty for a mining concern when the resource to be exploited is nonhomogeneous. Cortazar et al. (2001) evaluate the exploration, development, and extraction phases of a copper mine with investment timing flexibility, and open, close or abandonment decisions during the extraction phase. Lumley and Zervos (2001) consider the effect of switching costs in pausing and restarting 
a mining project. Similar to most of these papers, we study the option to pause production, i.e., scale the extraction rate. Different from these papers, we also investigate the interaction between this real option and the real options to scale the ET and CT levels in natural gas production.

ET and CT are discussed in detail in the natural gas and petroleum engineering literature, for example, by Coleman et al. (1991a - 1991c), Clegg et al. (1993), and Coşkuner and Strocen (2003). However, these authors do not quantify the economic value of optimally managing these technologies as real options, as we do in this paper. Moreover, we extend the commonly used models of natural gas production (e.g., Tarek 2006) to incorporate the effects of scaling the ET and CT levels on the flow rate evolution, consistent with the empirical observations available in the petroleum engineering literature (Coskuner and Strocen 2003).

The CT scaling option that we investigate is akin to the optimization of preventive maintenance decisions studied by Kamrad and Lele (1998) in a manufacturing environment with random yield and output prices. Unlike their model, in our model the scaling of CT is reactive and impacts the evolution of the extraction rate, not only the instantaneous production rate. Moreover, different from Kamrad and Lele (1998), we investigate the interaction of the CT scaling option with the ET scaling and production pausing options.

Our work is also related to the paper by Moel and Tufano (2002) who study opening and closing decisions of gold mines' managers as a function of industry and firm level characteristics. But their focus is descriptive, and their aim is to empirically test whether gold mines' managers opening and closing decisions are consistent with the basic predictions laid out in Brennan and Schwartz (1985). Our focus is prescriptive, and our aim is to bring to light model-based and data-driven principles that could be used by managers to inform their decisions on how to manage technology and extraction scaling options in the production of natural gas and other commodities. Moreover, Moel and Tufano (2002) do not include technology scaling decisions in their analysis. This is an important distinction as these scaling options significantly increase the value of the property and managing them optimally is not straightforward.

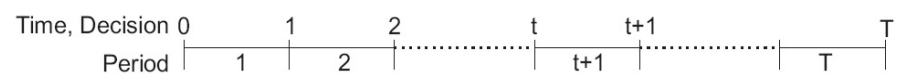

Figure 1 Timeline 


\section{Model}

In this section we present our stochastic dynamic programming model that optimizes technology and extraction scaling decisions during a finite time horizon. Let $\mathrm{T}$ denote the end of this time horizon, which is assumed to be divided into a set of equal length time periods (see Figure 1). We normalize this length to one. Even though we assume that the well's flow rate and the natural gas price evolve in continuous time, in our stochastic dynamic program decisions are made only at times $0,1, \ldots, T-1$, the start of time periods $1, \ldots, T$. (That is, the stages of this model correspond to the times $0,1, \ldots, \mathrm{T}$.) The state of our stochastic dynamic program at time $t$ is the vector $(p, q, x, y)$, which includes the natural gas spot price, $p \in \mathbb{R}_{+} ;$the well's flow rate, $q \in \mathbb{R}_{+} ;$and the ET and CT levels, $x$ and $y$. We assume that there are $X+1$ and $Y+1$ possible ET and CT levels, which belong to sets $\mathcal{X}:=\{0, \ldots, X\}$ and $\mathcal{Y}:=\{0, \ldots, Y\}$, respectively. Thus, the state space in any stage is the set $\mathcal{S}:=\left\{(p, q, x, y): p \in \mathbb{R}_{+}, q \in \mathbb{R}_{+}, x \in \mathcal{X}, y \in \mathcal{Y}\right\}$.

At time $\mathrm{T}$, if still operational, the well is abandoned (which could be required by contractual agreements). At each time $t<\mathrm{T}$, the production manager decides how to operate the well in time period $t+1$. Specifically, this manager decides whether to

1. Produce using the currently deployed technology.

2. Abandon the well and forego the opportunity of ever producing from it again. (When abandoning, all equipment at the well is removed.)

3. Pause production and leave all the equipment as is, to potentially resume production or abandon the well at a future time.

4. Invest or divest in $\mathrm{ET}$ and/or $\mathrm{CT}$, and either produce or pause during time period $t+1$.

We denote by $a:=\left(a_{1}, a_{2}, a_{3}\right)$ the action vector in a given state and stage. This vector specifies the new ET level, $a_{1}$, the new CT level, $a_{2}$, and whether to produce, $a_{3}=1$, pause, $a_{3}=0$, or abandon, $a_{3}=-1$. We denote the action set at time $t<\mathrm{T}$ by $\mathcal{A}(t):=\left\{\left(a_{1}, a_{2}, a_{3}\right): a_{1} \in \mathcal{X}, a_{2} \in \mathcal{Y}, a_{3} \in\right.$ $\{-1,0,1\}$. Decisions on the technology levels made at time $t<\mathrm{T}$ are immediately implemented, so the system in period $t+1$ operates at technology levels $a_{1}$ and $a_{2}$. This is reasonable because of the limited magnitude of the changes that need to be made to the well.

The cash flows in stage $t$ are determined by the gas price, $p_{t}$, and the flow rate, $q_{t}$, at time $t$, taking into account the instantaneous implementation of the decision $a$ at time $t$ and any cost associated with this decision. Since many gas (and oil) wells are located in remote areas, weather 
conditions can significantly impact their operational and investment costs. Therefore, the relevant costs in our model are time dependent. We consider the following cost factors:

- Preventive maintenance cost $c_{t}^{M}$ that is associated with basic seasonal maintenance jobs. This cost is paid in every stage up to abandonment.

- Operating cost $c_{t}^{O}(x, y)$ that depends on the currently employed technology levels $x$ and $y$. When a well is paused or abandoned, no operating cost in incurred.

- Investment cost $c_{t}^{I}\left(x, y, a_{1}, a_{2}\right)$, to change the technology levels from $(x, y)$ to $\left(a_{1}, a_{2}\right)$. We assume the investment cost is separable in technology type, i.e., $c_{t}^{I}\left(x, y, a_{1}, a_{2}\right)=c_{t}^{I E}\left(x, a_{1}\right)+$ $c_{t}^{I C}\left(y, a_{2}\right)$, where $c_{t}^{I E}\left(x, a_{1}\right)$ and $c_{t}^{I C}\left(y, a_{2}\right)$ are the costs of changing the ET level and CT level, respectively. The investment cost is positive when the technology level is increased and may be negative if the technology level is decreased, due to the salvage value of the equipment.

- Abandonment $\operatorname{cost} c_{t}^{A}$ that may be positive or negative depending on whether the well is sold or needs to be dismantled at no salvage value. When a well is abandoned, the installed ET and CT need to be dismantled at $\operatorname{costs} c_{t}^{I E}(x, 0)$ and $c_{t}^{I C}(y, 0)$, respectively.

The stochastic component of our model is the price process. We assume that the natural gas spot price evolves according to an exogenously specified stochastic process that is not affected by the flow rate evolution and the manager's operational decisions. This is consistent with other models used in the literature (e.g., Smith and McCardle 1998). In our numerical analysis in $\S 5$ and $\S 6$ we use the seasonal mean reverting price process of Jaillet et al. (2004). Schwartz (1997) and Seppi (2003) provide excellent reviews of the typical reduced-form models of the evolution of commodity prices that are employed in the real option literature.

We model the well's flow rate as a deterministic process. Although other authors, such as Smith and McCardle (1998), have used stochastic models of flow rate evolution, our choice is motivated by modeling simplicity, i.e., it allows us to capture the effect of technology scaling on the flow rate evolution in a parsimonious fashion, as discussed below. In addition the deterministic model corresponds well with models used in practice, as discussed below. We leave the extension to a stochastic case of our model to future research, as pointed out in $\S 7$.

The flow rate at time $t+1, q_{t+1}$, with $t<\mathrm{T}$, depends on the time $t$ flow rate, $q_{t}$, and the action vector, $\mathcal{A}(t)$. If the well is abandoned then $q_{t+1}$ is set equal to 0. Otherwise, if ET and CT are unmodified, then $q_{t+1}$ is equal to $q_{t}$ if the well is paused, and changes to $q_{t} \exp [-\mu(x, y)]$ if the well is producing, where $\mu(x, y)>0$ is the technology dependent decline rate of the well's flow rate. 


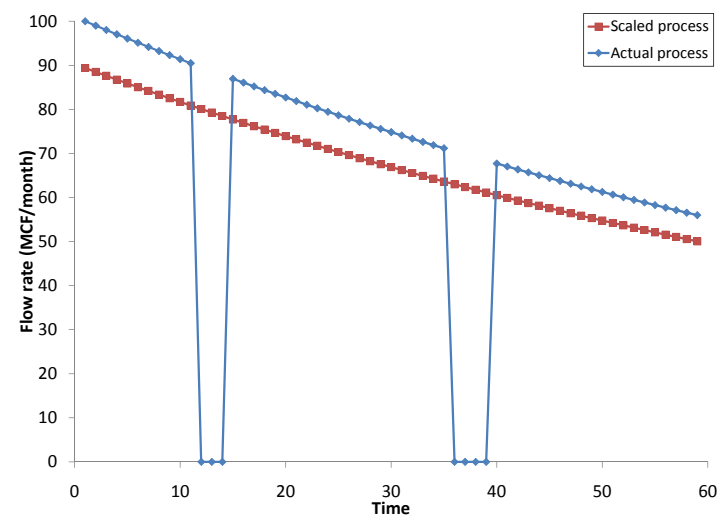

Figure 2 Sketch of the actual and scaled flow rate processes.

When the ET and CT technology levels are unchanged, this model of exponential flow rate decline is commonly used in the petroleum engineering literature (see, e.g., Tarek 2006); in particular, the positive decline rate is consistent with the behavior of mature wells, as we model here. If the well is not abandoned, modifying either one of the current ET or CT levels affects both $q_{t}$ and $\mu(x, y)$. We capture these effects by embedding petroleum engineering models of natural gas well liquid load-up behavior (Clegg et al. 1993 and Coleman et al. 1991a-1991d) into the exponential flow rate decline model.

We introduce the notion of nominal flow rate, denoted by $\bar{q}_{t}$. This is the flow rate at an idealized CT level that would ensure a production yield equal to $100 \%$. For ET level $x$, the nominal flow rate evolves according to the exponential model with decline rate $\bar{\mu}(x)$ (we discuss how we model this rate below). We relate the flow rate $q_{t}$ to the nominal flow rate $\bar{q}_{t}$ by using the production yield $\rho(y) \in(0,1)$, which depends on the employed CT level $y$. Specifically, if the time $t$ nominal flow rate is $\bar{q}_{t}$ and the ET and CT levels are $x$ and $y$, then the flow rate and its decline rate satisfy the identities $q_{t} \equiv \rho(y) \bar{q}_{t}$ and $\mu(x, y) \equiv \rho(y) \bar{\mu}(x)$. This means that our representation of the flow rate is a smoothed version of an underlying flow rate model with load-up events (see Figure 2).

We model the production yield $\rho(\cdot)$ as follows. CT affects how quickly one can respond to a situation in which a well is loaded up with liquid. Wells that are operational are assumed to load-up according to a geometrically distributed time to failure with mean $1 / \lambda$, where $\lambda>0$. Loaded-up wells are assumed to return to operational status through corrective maintenance, i.e., recover, with a geometrically distributed time with mean $1 / \eta(y)$, where $\eta(y)>0$ depends on the employed CT level $y$. We define the production yield at CT level $y$ as the fraction of time that a well is operational: $\rho(y):=1 /[1+\lambda / \eta(y)]$. We assume that this quantity increases in the CT level $y$ by 
assuming that $\eta(y)$ does so, which is natural, i.e., the mean recovery time decreases in the CT level $y$.

We now discuss the effect of changing the ET and CT levels from $(x, y)$ to $\left(a_{1}, a_{2}\right)$ at time $t$, when the flow rate is $q_{t}$. Using the identities that relate the flow rate to the nominal flow rate and their decline rates, the CT change from level $y$ to level $a_{2}$ has two effects: (i) it modifies the flow rate from $q_{t}$ to $\rho\left(a_{2}\right) q_{t} / \rho(y)$, as $q_{t} / \rho(y)$ is the nominal flow rate corresponding to $q_{t}$; (ii) it modifies the decline rate from $\mu(x, y) \equiv \rho(y) \bar{\mu}(x)$ to $\rho\left(a_{2}\right) \bar{\mu}(x)$. Notice that if $a_{2}>y$, then this CT change increases the rate of decline of the flow rate; this implies that the new flow rate also increases.

The ET change from level $x$ to level $a_{1}$ affects both the flow rate and its decline rate. Notice that if $a_{1}>x$, then this change instantaneously raises the flow rate and reduces its decline rate; this is consistent with the empirical observations made by Coşuner and Strocen (2003), which are reflected in our data set, as discussed in $\S 4$. We use the given function $\delta\left(x, a_{1}\right)$, with $\delta\left(x, a_{1}\right)>1$ for $x<a_{1}$, to model the effect on the flow rate $q_{t}$ of changing the ET level from $x$ to $a_{1}$ as follows: $q_{t}$ immediately changes to $\delta\left(x, a_{1}\right) q_{t}$. To model the effect on the decline rate of changing the ET level from $x$ to $a_{1}$, we first must model the decline rate of the nominal flow rate $\bar{\mu}(0)$. We use the function $\gamma(x)$ for this purpose: We assume that $\gamma(0)=1$ and that $\gamma(\cdot)$ is an increasing function. We then define the decline rate of the nominal flow rate at ET level $x$, relative to its value at level 0 , as $\bar{\mu}(x):=\gamma(x) \bar{\mu}(0)$. Changing the ET level from $x$ to $a_{1}$ changes the decline rate of the nominal flow rate from $\gamma(x) \bar{\mu}(0)$ to $\gamma\left(a_{1}\right) \bar{\mu}(0)$. Thus, this change modifies the decline rate of the flow rate from $\rho(y) \gamma(x) \bar{\mu}(0)$ to $\rho(y) \gamma\left(a_{1}\right) \bar{\mu}(0)$.

We aggregate the effects of changing technologies through the flow rate transition function $f_{t}\left(q_{t}, x, y, a\right)$; this function yields the flow rate at time $t+1: q_{t+1}=f_{t}\left(q_{t}, x, y, a\right)$. We define this function as follows:

$$
f_{t}\left(q_{t}, x, y, a\right):= \begin{cases}0 & \text { if } a_{3}=-1, \\ \delta\left(x, a_{1}\right) \frac{\rho\left(a_{2}\right)}{\rho(y)} q_{t} & \text { if } a_{3}=0, \\ \delta\left(x, a_{1}\right) \frac{\rho\left(a_{2}\right)}{\rho(y)} \exp \left[-\rho\left(a_{2}\right) \gamma\left(a_{1}\right) \bar{\mu}(0)\right] q_{t} & \text { if } a_{3}=1 .\end{cases}
$$

If the well is abandoned the flow rate drops to 0 ; otherwise, the flow rate immediately changes to $\delta\left(x, a_{1}\right) \rho\left(a_{2}\right) q_{t} / \rho(y)$ and stays at this level if the well is paused, and declines exponentially from this level with decline rate $\rho\left(a_{2}\right) \gamma\left(a_{1}\right) \bar{\mu}(0)$ if the well produces during the next period.

We now formulate our stochastic dynamic program. We assume that there exists a natural gas futures market, e.g., NYMEX, that is arbitrage free and complete. Thus, we employ a risk-neutral 
valuation approach (Luenberger 1998, Smith 2005). We use the one period risk free discount factor $\alpha$. We let $\tilde{p}_{t}$ be the random variable denoting the natural gas price at time $t$. We denote by $1\{\cdot\}$ the indicator function that is 1 if its argument is true and 0 otherwise. We let $r_{t}(p, q, x, y, a)$ denote the following immediate payoff function associated with action $a \in \mathcal{A}(t)$ in state $(p, q, x, y) \in \mathcal{S}$ at time $t=0, \ldots, \mathrm{T}-1$ :

$$
r_{t}(p, q, x, y, a):= \begin{cases}-c_{t}^{I}\left(x, y, a_{1}, a_{2}\right)-c_{t}^{A} & \text { if } a_{3}=-1, \\ -c_{t}^{M}-c_{t}^{I}\left(x, y, a_{1}, a_{2}\right) & \text { if } a_{3}=0, \\ -c_{t}^{M}-c_{t}^{I}\left(x, y, a_{1}, a_{2}\right)-c_{t}^{O}\left(a_{1}, a_{2}\right)+p \delta\left(x, a_{1}\right) \frac{\rho\left(a_{2}\right)}{\rho(y)} q & \text { if } a_{3}=1 .\end{cases}
$$

The optimal value function of our model, $V_{t}$, satisfies the following Bellman equations:

$$
\begin{aligned}
V_{t}(p, q, x, y) & =\max _{a \in \mathcal{A}(t)} v_{t}(p, q, x, y, a), \forall t=0,1, \ldots, \mathrm{\top}-1,(p, q, x, y) \in \mathcal{S} \\
v_{t}(p, q, x, y, a) & :=r_{t}(p, q, x, y, a)+1\left\{a_{3} \neq-1\right\} \alpha \mathbb{E}\left[V_{t+1}\left(\tilde{p}_{t+1}, f_{t}(q, x, y, a), a_{1}, a_{2}\right) \mid p_{t}\right] \\
V_{\mathrm{\top}}(p, q, x, y) & :=-c_{\mathrm{T}}^{I}(x, y, 0,0)-c_{\mathrm{\top}}^{A}, \forall(p, q, x, y) \in \mathcal{S} .
\end{aligned}
$$

\section{Data}

In this section we discuss how we, in cooperation with production managers at EQT Corp., assessed the costs of operating our subject natural gas well located in Eastern Kentucky owned and operated by EQT Corp., the costs of investing and divesting in technology, and the well performance parameters. We focus our attention on this particular well as it provides insights into the effect of technology change. We also discuss the estimation of the parameters of the natural gas price process.

Our well was drilled in the late 1950's. Although the flow rate of wells may show non-exponential decline behavior early in a well's life cycle (Garb and Larson 1989), we can safely assume our well has entered its phase of exponential flow rate decline. Our well was operated as a continuous flow well until 2003; in January 2003 it was equipped with soap and blow technology. (These types of ET technologies are described in $\S 1$.) We let 0 and 1 denote the continuous-flow and soap-and-blow ET levels, respectively. Data on the well's operational status has always been obtained periodically; we let this data collection approach be CT level 0 . The possibility of employing an automated and continuous data collection approach, which we indicate by CT level 1, was being investigated at EQT Corp. at the time of this study.

Figure 3 shows monthly production data from the well from 1986 to 2009 (MCF = one thousand cubic feet). After the technology change in January 2003, the flow rate of our well increased and 


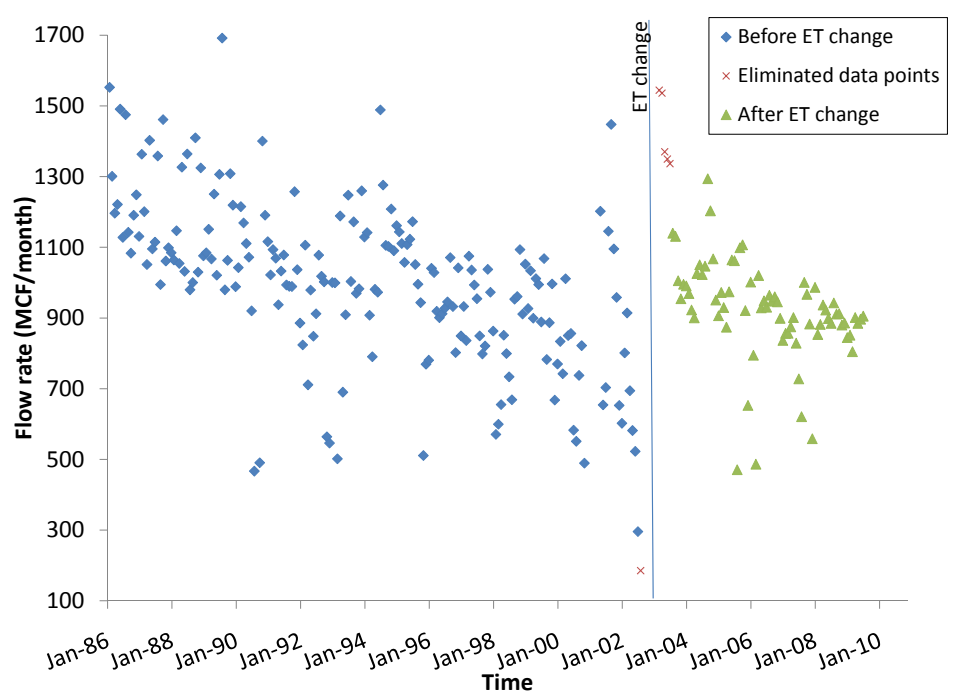

Figure 3 Historical (monthly) production data for the well studied in this paper.

its decline rate decreased, which is consistent with our flow rate modeling approach discussed in $\S 3$. We used this data to estimate the parameters of our well flow rate evolution model.

After consultation with production managers at EQT Corp. we eliminated production rates of zero because they presumably originated from a failure of the measurement device. We also excluded "very low" production rates, two data points before the technology change, because they were deemed part of irregular well behavior caused by external factors. In addition, the well became unoperational for the five months before the change in technology. This led to a build-up of pressure and thus likely caused elevated production rates immediately after the well was brought back online. Thus, we eliminated the first five exceptionally high production rates after production resumed.

We estimated the parameters of the process describing the flow rate evolution using linear regression on the natural logarithms of the flow rate data. The estimate for $\mu(0,0)$ is $8.32 \%$ per year, and that of $\mu(1,0)$ is $3.19 \%$ per year; hence $\gamma(1)=0.38$. Thus upgrading ET from continuous flow to soap and blow leads to significant operational improvement.We averaged the twelve retained data points immediately before and after the ET change in January 2003 to estimate the flow rate before and after this change. These values are 27.8 MCF/Day and 33.36 MCF/Day, which provides an estimate of 1.20 for $\delta(0,1)$. The estimate is consistent with the observations of Coskuner and Strocen (2003), on which our model of flow rate behavior is based.

The production data from our example well did not have high enough granularity to estimate the yield function $\rho(\cdot)$, so we used up to 50 years of production data for a set of 135 of EQT 
Table 1 Cost parameters for summer (winter).

\begin{tabular}{ll||l|l|} 
& \multicolumn{1}{c||}{} & \multicolumn{2}{c|}{ CT (monitoring frequency) } \\
& & Periodic (0) & Continuous (1) \\
\hline \hline \multirow{2}{*}{ ET Continuous flow (0) } & $100(105)$ & $110(115.5)$ \\
& Soap and blow (1) & $250(262.5)$ & $275(288.8)$ \\
\hline
\end{tabular}

(a) Operating cost $(\$ /$ month).

\begin{tabular}{l||l|l|} 
& ET & CT \\
\hline \hline Investment $(0 \rightarrow 1)$ & $1,000(1,050)$ & $8,000(8,400)$ \\
Divestment $(1 \rightarrow 0)$ & $300(315)$ & $-2,667(-2,540)$ \\
\hline
\end{tabular}

(b) Technology investment and divestment costs $(\$)$.

Corp.'s soap and blow wells to estimate the yield. We identified periods during which these wells were loaded up and estimated $\rho(0)$ as the average fraction of time that the wells were producing, i.e., we directly estimated the quantity $1 /[1+\lambda / \eta(0)]$. This leads to an estimate for $\rho(0)$ of $90 \%$. As no data was available on the effect of continuous monitoring, we assumed $\rho(1)$ to be equal to 99\%, as this CT level would enable a more timely resolution of liquid load-up situations.

The relevant costs were estimated using various sources within EQT Corp. For confidentiality, these costs do not specifically represent those of our well, but are representative for this type of well. We distinguish between summer (April through October) and winter (November through March). Costs during the summer are as follows (winter costs are $5 \%$ higher): The maintenance cost is $\$ 321$ per month. The abandonment cost is $\$ 36,250$. The cost of operating the well is $\$ 100$ per month with continuous flow ET and $\$ 250$ per month with soap and blow ET, both at the lowest CT level, i.e., periodic monitoring. We estimated a 10\% higher operating cost with continuous monitoring. The investment cost to move from continuous flow to soap and blow is $\$ 1,000$. The divestment cost to move from soap and blow to continuous flow is $\$ 300$. The CT that enables continuous monitoring costs $\$ 8,000$ to install and has a salvage value of $\$ 2,677$. Table 1 summarizes these costs (the winter cost are in parentheses).

We use NYMEX natural gas futures prices and prices of options on natural gas futures traded on $5 / 29 / 2009$ to estimate the parameters of the price model of Jaillet et al. (2004). Figure 4 displays the NYMEX natural gas forward curve on this date, along with the fit of our model. This curve exhibits significant seasonality. The model of Jaillet et al. (2004) features twelve deterministic monthly seasonality factors and a deseasonalized spot price, whose natural logarithm evolves as a single factor mean reverting process. The parameters of this model are the current level of this factor and its volatility, speed of mean reversion, and long term level; this last parameter is however not needed for valuation purposes (Jaillet et al. 2004). We used the method described by Lai et al. (2009) to estimate these parameters. This yielded an estimate of the natural gas spot price on 


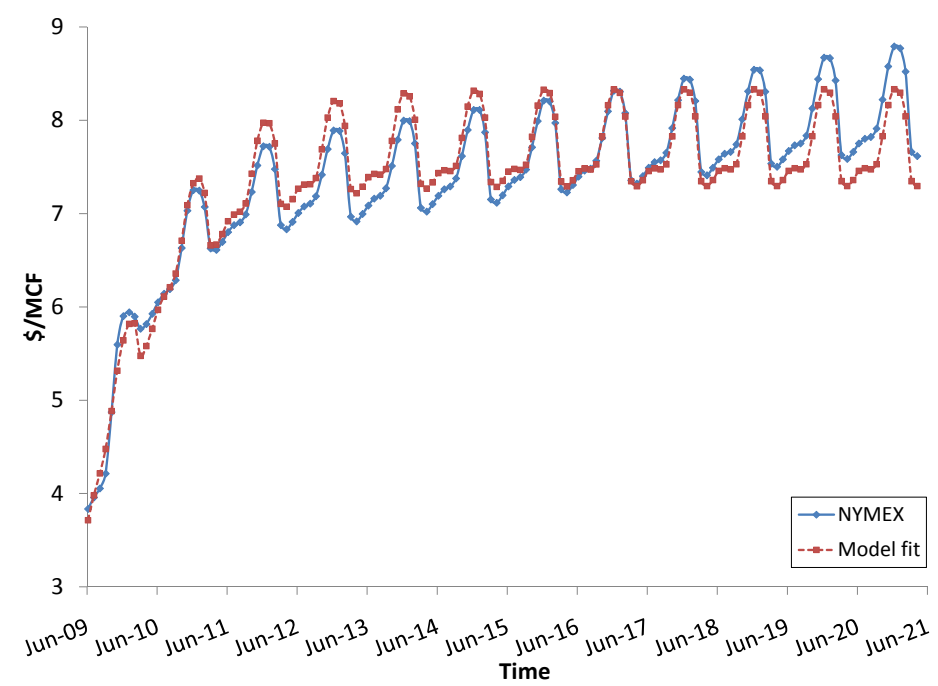

Figure 4 NYMEX natural gas forward curve on 5/29/2009 and model fit.

$5 / 29 / 2009$ equal to $\$ 3.38$ (the actual spot price was $\$ 3.92$ ), estimates of the volatility and speed of mean reversion parameters equal to $67 \%$ and 1.05, and estimated seasonality factors (displayed in Table 2) ranging between 0.946 (May) and 1.081 (January).

Table 2 Seasonality factors of the natural gas price.

\begin{tabular}{|r||l|l|l|l|l|l|}
\hline Month & Jan & Feb & Mar & Apr & May & Jun \\
Seasonality factor & 1.081 & 1.076 & 1.043 & 0.953 & 0.946 & 0.955 \\
\hline Month & Jul & Aug & Sep & Oct & Nov & Dec \\
Seasonality factor & 0.967 & 0.971 & 0.970 & 0.977 & 1.016 & 1.059 \\
\hline
\end{tabular}

\section{Valuation Results}

In this section we discuss the scaling option valuation results obtained by applying our stochastic dynamic program to the data described in $\S 4$ (we do not discuss the value of the abandoning option as our focus is on the technology and extraction scaling options).

We consider a 100-year horizon, with monthly stages. We use an annualized risk free discount rate of $5 \%$. We implement a discrete time and space version of the price evolution model using a trinomial tree constructed by applying the method described by Jaillet et al. (2004), using monthly time intervals and the estimated natural gas spot price on 5/29/2009 as the initial price level. As the natural gas forward curve on 5/29/2009 only includes 144 monthly maturities, we reuse the last 12 prices in this forward curve to generate a 100 year forward curve that we use to calibrate this trinomial tree. 
We also implement a discrete time and space version of the flow rate evolution, because modifications to the flow rate due to pausing and technology changes preclude the use of a single decline curve. We set the starting flow rate level equal to $27.8 \mathrm{MCF} /$ Day; this is the average flow rate level of the 12 retained data points immediately preceding the ET level change discussed in $\S 4$. We use a grid in which the natural logarithms of the flow rate at the start of each month are equally spaced. The lowest flow rate considered is $0.001 \mathrm{MCF} /$ Day, and each next value on the grid is a $1 \%$ increase on the previous. The largest value on the grid for the ET level 0 is $30.59 \mathrm{MCF} / \mathrm{Day}$, which is the smallest grid value that exceeds $27.8 \mathrm{MCF} /$ Day multiplied by $0.99 / 0.90$ (as the time $0 \mathrm{CT}$ level is 0). For the ET level 1 the largest value on the grid is $36.96 \mathrm{MCF} /$ Day, which is the smallest entry on the grid that exceeds 30.59 MCF/Day multiplied by 1.20. If transitioning from one stage to the next according to (1) leads to a flow rate that is not on the grid, we linearly interpolate between the optimal value functions corresponding to the adjacent grid values.

The value of an option is the difference between the optimal value functions of our model in the initial stage, given the initial flow rate and price, with and without this option as part of the available decisions. The option value of a portfolio of options is calculated in a similar manner. We consider both the intrinsic and total (intrinsic plus extrinsic) values of an option. The former is the option value when the natural gas spot price evolves deterministically (approximated by setting the volatility parameter equal to 0.00001 ). The latter includes the effect of positive price volatility, i.e., set to its estimated value discussed in $\S 4$.

We find it useful to display our results using the cubes in Figure 5. The three axes of each cube correspond to the availability of an option (at -1 an option is not available, at +1 it is). Panels $(a)$ and $(b)$ of Figure 5 show the intrinsic and total option values, respectively, measured as percent increases from the base value of $\$ 445,478$. This is the value of the well when (i) it cannot be paused, (ii) no technology is available, and (iii) there is no price volatility. We select this as the base case because it allows us to distinguish between extrinsic and intrinsic values, and it yields the lowest value function for our model, thus making all the considered option values positive.

We discuss the option valuation results in detail in $\S \S 5.1-5.3$, and provide a summary of our valuation results in $\S 5.4$. We point out that the real option valuations and managerial insights that we discuss are specific to the data that we use. 


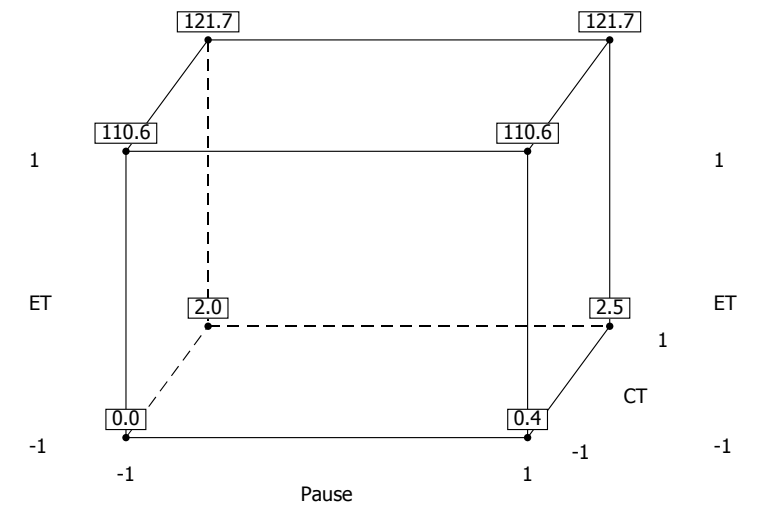

(a) Percent intrinsic value increase

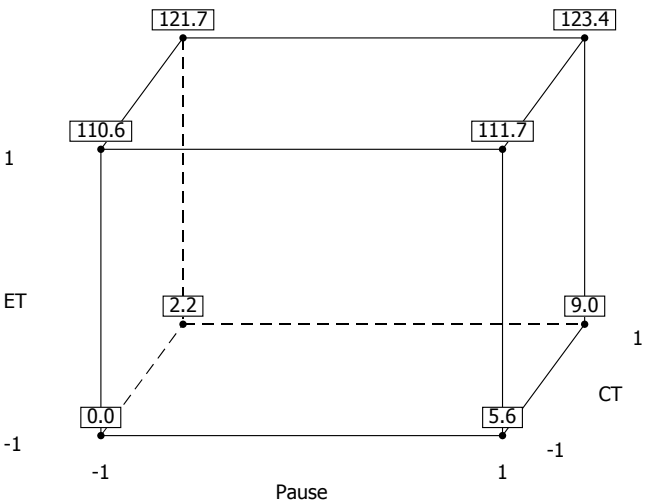

(b) Percent total value increase

Figure 5 Valuation results; the displayed figures are percent increases over the well's intrinsic value without scaling options $(\$ 445,478)$

\subsection{Technology Scaling Options}

The left vertices of the cubes in Figure 5 display the valuation results of the technology scaling options, in isolation from the extraction scaling option; i.e., pausing is not available.

The ET scaling option has the largest total option value, increasing the well's total value by $110.6 \%$, while the CT scaling option yields a $2.2 \%$ increase in total value (see Figure 5(b)). The value of the ET scaling option is entirely intrinsic. This value is obtained through access to larger reserves - recall that the flow rate level increases and its decline rate decreases when the ET level increases. The value of the CT scaling option exhibits very little sensitivity to price volatility: its intrinsic value is $2.0 \%$ in Figure $5(\mathrm{a})$, and its total value is $2.2 \%$ in Figure $5(\mathrm{~b})$. As CT affects the speed at which one extracts natural gas, the high CT level allows one to produce at a higher rate when prices are high; conversely the low CT level allows one to reduce production, i.e., save natural gas, when prices are low. However, the additional value brought about by this flexibility in the presence of price volatility is minimal.

The total value of the ET scaling option in the presence of the CT scaling option increases from $110.6 \%$ to $121.7 \%-2.2 \%=119.5 \%$. The total value of the CT scaling option in the presence of the ET scaling option increases from $2.2 \%$ to $11.1 \%$. Thus, the two technology scaling options can be seen as complements: Considering both options simultaneously adds $121.7 \%$ to the well's total value, $8.9 \%$ more than the sum of the two options' individual values. As both technology options yield a percent increase in the immediate flow rate, their complementarity stems from the benefit 
of adding a percent increase to a higher flow rate, i.e., the flow rate obtained after increasing the level of one of the two technologies.

\subsection{Extraction Scaling Option}

The front, bottom vertices of the cubes in panels $(a)$ and $(b)$ of Figure 5 display the values of the extraction scaling option; i.e., pausing without any technology option. Pausing has little value without price volatility: The total value of this option is $5.6 \%$, compared with an intrinsic value of 0.4\%. Pausing can be used to delay production from a well during periods with low prices (it has other uses as well; see Example 3 in $§ 6.1)$; i.e., to "save up gas" by postponing production until the price rises. Thus, pausing can be interpreted as a storage option, used when prices are low, which also saves operating costs. However, deterministic variations in prices, as expressed by the marked seasonality of the natural gas forward curve (see Figure 4 in $\S 4$ ), are not sufficient for this storage option to have significant value, which instead requires stochastic price variability.

\subsection{Portfolio of Technology and Extraction Scaling Options}

The right, top, and rear vertices of the cubes in Figure 5 display the option value of a portfolio of technology and extraction scaling options. Lowering the CT level and pausing have a similar function, i.e., they help "save up" natural gas, but pausing also saves the operating cost and does not require capital outlays. Given their similarity one would expect these options to behave as substitutes. However this is not the case here. Combining pausing with the CT scaling option increases the total value of the pausing option from $5.6 \%$ to $6.8 \%$ and the total value of the CT scaling option from $2.2 \%$ to $3.4 \%$. The CT scaling and pausing options can hence be seen as complements, as their combined value of $9.0 \%$ exceeds the sum of their individual values, $7.8 \%$.

This is consistent with the following observations. When the CT scaling option is available, operating CT at its high level increases the decline rate of the well's flow rate. This exhausts the well sooner, which extends the amount of time during which pausing can be used without the wasting of production (the interval between the time the well is exhausted and the end of the horizon or the time when financial discounting renders production virtually worthless). Conversely, pausing can be used to limit the costly reduction of the CT level, i.e., from its high to its low level, whenever the well is operated at the high CT level and this reduction would be otherwise beneficial, e.g., when prices are low (recall that the salvage value of the continuous monitoring technology is significantly lower than its associated initial capital outlay). 
Using the pausing option in conjunction with the ET scaling option has an opposite effect. The total values of these options when used in isolation are $5.6 \%$ and $110.6 \%$, respectively. However, once these options are jointly used their total values decrease to $1.1 \%$ and $106.1 \%$, respectively. The pausing and ET scaling options can hence be seen as substitutes. This substitution effect can be explained as follows. Deploying ET at its high level increases the immediate flow rate and decreases its rate of decline. When the flow rate is sufficiently high, as it appears to be in our computational experiments, using the pausing option in conjunction with the high ET level would indeed amount to wasting production, by postponing it until much later in the horizon.

When we consider pausing in conjunction with the two technology scaling options, the substitution effect between pausing and the ET scaling option dominates the complementarity effect between pausing and the CT scaling option, as the total value of pausing in the absence of ET and $\mathrm{CT}, 5.6 \%$, is higher than its total value in the presence of these two options, $1.6 \%$. Hence, the pausing option and the portfolio that includes the two technology scaling option are substitutes.

Comparing the relevant vertices of the cubes in Figure 5 indicates that there is a complementarity relationship between each technology scaling option and each pair of the other two options. This reinforces our previous finding that the technology scaling options are complements. It also suggests that the complementarity between these options is stronger than the substitution relationships previously described.

Finally, we observe that the total option value of the portfolio that includes all the three scaling options, $123.4 \%$, whose intrinsic value is $121.7 \%$, is higher than the $110.6 \%+2.2 \%+5.6 \%=118.4 \%$ increase brought about by employing the three options individually. Although this result is implied by the complementarity between the CT scaling and pausing options and the complementarity between the ET scaling option and the portfolio that includes the two other scaling options, its magnitude is notable.

\subsection{Summary}

We summarize our valuation results as follows: When used in isolation, the ET, CT, and extraction scaling options increase the value of the well by $110.6 \%, 2.2 \%$, and $5.6 \%$, respectively. The relative values of these options reflect their usages: Exercising the ET scaling option increases the size of the extractable reserve, a first order effect; exercising the CT scaling or the pausing options improves operational efficiency and effectiveness, respectively, second order effects. Our finding 
on the value of pausing contrasts with current practice, where this option is typically equated to wasting production, and thus avoided if possible. The values of the technology scaling options are almost entirely intrinsic, while the value of pausing is almost fully extrinsic. Individual options and the portfolio that includes the two other relevant options are also complements, excluding the ET scaling option and portfolio that consists of the CT scaling and pausing options. Likewise, the option value of the portfolio that includes all the three scaling options exceeds the sum of the values of the individual options.

\section{Deployment Policies}

In this section we study how to effectively deploy the technology and extraction scaling options together with the abandonment option; i.e., we analyze the optimal exercise of these options. We begin in $\S 6.1$ by studying the optimal deployment results of our model numerically, finding that the optimal deployment of the three scaling options is complicated. This prompts us to explore a simpler - more practical - approach to their deployment in $\S 6.2$, which captures most of their combined value.

\subsection{Optimal Deployment Policy}

In this subsection we illustrate numerically that optimally deploying the three scaling options is rather complicated. We consider the same setting as in $\S 5$. Regarding the optimal deployment of the three scaling options, one might expect:

1. The optimally employed ET and CT levels to increase in the gas price and the flow rate; and

2. Pausing to be used primarily when prices are low (Smith and McCardle 1998, p. 211, and our discussion in $\S 5.2)$.

However, this is not true in general, as shown in Examples 1-4 below. These examples are based on Figure 6, which illustrates the optimal deployment of the technology and extraction scaling options, as well as the abandonment option, in different time periods as a function of the flow rate and the natural gas price. The labels associated with each region of this figure indicate the type of optimal action in each such region, and also indicate the optimal deployed levels of ET and $\mathrm{CT}$ as the pair $\{x, y\}$. Figures 6(a) and 6(b) pertain to January and April, respectively, but they are also representative of the other winter and summer months indicated; Figure 6(c) is October. For ease of interpretation, Figure 6 uses log scales, but the labels and the tickmarks are in the original units to facilitate intuition about the magnitudes displayed. In these examples the initial technology level pair is $\{1,0\}$. Similar results are obtained with different initial technology levels. 


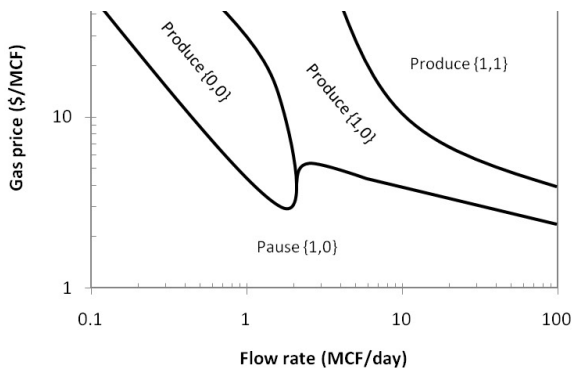

(a) January - March, November,

December.

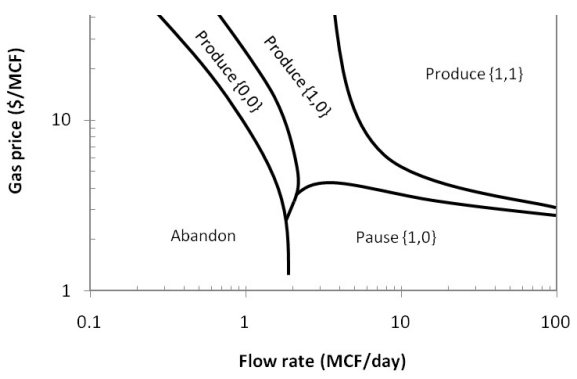

(b) April - September.

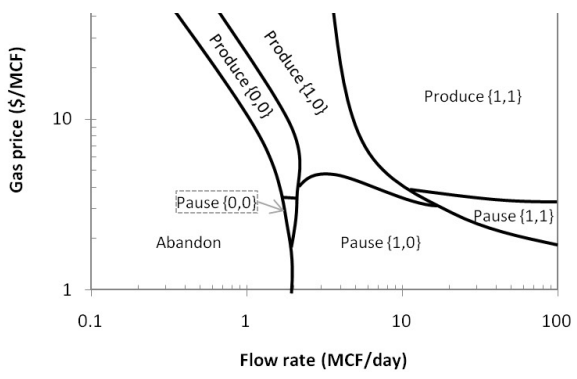

(c) October.

Figure 6 Optimal technology deployment and operating decisions in different stages as functions of the flow rate and the natural gas price with the ET level equal to 1 and the CT level equal to 0 . The captions of the panels indicate the period(s) for which the panel is representative: the month pertaining to the displayed panel is italicized.

Example 1. Consider any flow rate below $2 \mathrm{MCF} /$ Day in Figure 6(a). At this flow rate, the optimally deployed ET level is nonmonotonic in the natural gas price (a similar behavior occurs for the CT level when the starting technology level pair is $\{0,1\})$.

ExAmple 2. Consider any price above $3 \$ / \mathrm{MCF}$ in Figure $6(\mathrm{a})$. At this natural gas price, the 
optimally deployed ET level is nonmonotonic in the flow rate (we observe a similar behavior for the CT level when the starting technology level pair is $\{0,1\})$.

ExAmple 3. Consider a price roughly equal to $3 \$ / M C F$ in Figure $6(a)$. At this natural gas price, the optimal pausing decision is nonmonotonic in the flow rate. For gas prices near this level it is optimal to pause at low flow rates, produce at flow rates between 1 and $2 \mathrm{MCF} /$ Day and at very high flow rates, while it is optimal to pause for flow rates between 2 and $30 \mathrm{MCF} / \mathrm{Day}$. At low flow rates, and even for high natural gas prices, pausing is used to delay abandoning until summer, as shown in Figure 6(b), when abandoning is cheaper. (At high flow rates pausing is used to save up gas, as discussed in §5.2.) This exemplifies how the seasonality of the cost structure affects the optimal policy. This is further illustrated in Example 4.

Example 4. Figure 6(c) shows that in the "Pause $\{0,0\}$ " and "Pause $\{1,1\}$ " regions it is optimal to change one of the technology levels, which are initially at the $\{1,0\}$ levels, and delay production at the new technology level. This occurs because in winter, which starts in November, the cost structure is less favorable than in summer.

\subsection{Heuristic Deployment Policy}

Given the complex structure of optimally deploying the three scaling options, we seek a simpler, but still effective approach to their deployment. We start by studying the benefit of actively managing the deployment of the technology scaling options over time, comparing our valuation results discussed in $\S 5$ with those obtained by running our model by fixing ET and CT at given levels throughout the time horizon. Figure 7 displays the relevant total value increases relative to the base case. Note that we still actively manage the pausing and abandonment options (the former option when it is available).

Comparing the cubes in Figures 5(b) and 7 reveals that actively managing the technology scaling options yields only a very small increase in the value of the well. Specifically, only in the absence of the ET scaling and the pausing options is there a positive difference (for the CT scaling option) in valuations, and even in this case the difference is very small. Hence, essentially all of the value increase brought about by using an optimal deployment policy can be attained by the following simpler policy: (i) exercise the two technology options immediately by switching to their high levels, and (ii) optimally manage the pausing and abandonment options thereafter. Though not explicitly illustrated here, we also expect this policy simplification to be valuable when managing a portfolio in which a larger set of technology levels is considered. 


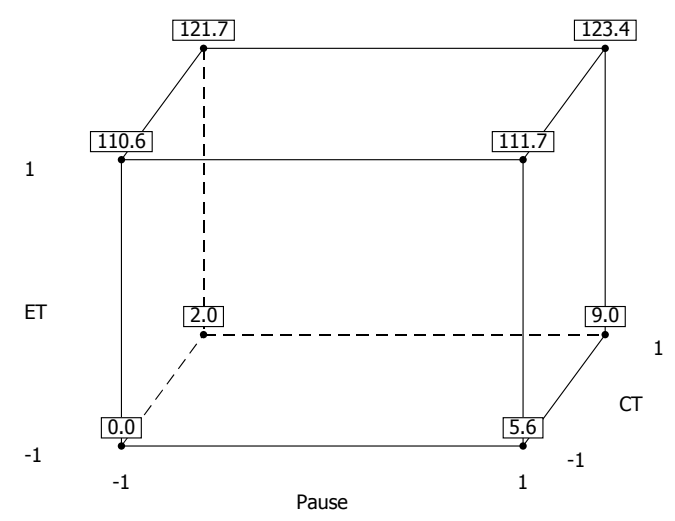

Figure 7 Valuation results with fixed technology scaling options; the figures displayed are the percent total value increases relative to the base case $(\$ 445,478)$.

\section{Conclusions}

In this paper we study the interaction between technology and extraction scaling options in natural gas production by developing and applying a stochastic dynamic program that uses empirical production and financial data. Our research is grounded in practice; it was developed in conjunction with production managers at EQT Corp. We bring to light basic managerial principles related to the drivers of these options' values and their deployment. These principles are likely to have managerial relevance in other commodity production settings, such as oil extraction and mining.

Our work has the potential to be extended in several dimensions. We employ a single-factor mean reverting model to describe the evolution of the price of natural gas. Although mean reversion in commodity prices is well documented in the literature (Clewlow and Strickland 2000, Chapter 2, Smith and McCardle 1999), more elaborate, multifactor models of commodity price evolutions are also available, such as the two-factor reduced-form and equilibrium models of Schwartz and Smith (2000) and Routledge et al. (2000), respectively. Our model uses a deterministic representation of the evolution of the flow rate of a natural gas well. It would be interesting to extend our work by using a stochastic model of production, as is often done in the real option literature (see, e.g., Smith and McCardle 1998). This extension might benefit from the application of the method described by Hahn and Dyer (2008) to model the discrete time and space evolution of correlated mean reverting processes. In addition, our analysis is based on data pertaining to a specific natural gas well; it would be of interest to examine the dependence of our conclusions on this data by replicating our analysis based on data from other wells.

More broadly, one could extend our work to consider the strategic management of the operations 
of a natural gas field, which includes multiple wells. This presents additional research opportunities related to modeling the transfer of technology across wells, the dependence among the flow rates of different wells, and the staffing and routing of maintenance operators. Moreover, when a production firm has a pre-committed production level and operates a gas field whose wells' flow rates are subject to natural decline, there may be multiple options to maintain production at the precommitted level, including drilling more wells. Our research suggests technology deployment as an alternative approach to meet the pre-committed production level. It would be interesting to evaluate the financial effect of this flexibility. This would require an even more complete well and operations model to asses the trade-offs between investing in current wells or drilling new ones. Such a strategic model could start at the pre-drilling phase, in which case the initial investment costs may depend on well characteristics that may only be discovered after drilling occurs.

\section{Acknowledgments}

The authors gratefully acknowledge the support of and discussions with Fil Sciullo, Tim Dugan, and Stuart Olson at EQT Corp. Furthermore, we would like to thank Fallaw Sowell and Sunder Kekre for their valuable comments and suggestions. The constructive feedback of the Department Editor, Jeremy Staum, and the review team led to significant improvements to this paper, and is gratefully acknowledged.

\section{References}

Al-Harthy, M.H. 2007. Stochastic oil price models: comparison and impact. The Engineering Economist 52(3) 269-284.

Brennan, M.J., E.S. Schwartz. 1985. Evaluating natural resource investments. Journal of Business 58(2) $135-157$.

Clarke, H.R., W.J. Reed. 1990. Oil-well valuation and abandonment with price and extraction rate uncertain. Resources and Energy 12(4) 361-382.

Clegg, J.D., S.M. Bucaram, N.W. Hein. 1993. Recommendations and comparisons for selecting artificial-lift methods. Journal of Petroleum Technology 45(12) 1128-1131, 1163-1167.

Clewlow, L., C. Strickland. 2000. Energy Derivatives: Pricing and Risk Management. Lacima Publications, London, UK.

Coşkuner, G., T. Strocen. 2003. Production optimization of liquid loading gas condensate wells: A case study. Journal of Canadian Petroleum Technology 42(11) 40-44. 
Coleman, S.B., H.B. Clay, D.G. McCurdy, H.L. Norris III. 1991a. Applying gas-well load-up technology. Journal of Petroleum Technology 43(3) 344-349.

Coleman, S.B., H.B. Clay, D.G. McCurdy, H.L. Norris III. 1991b. A new look at predicting gas-well load-up. Journal of Petroleum Technology 43(3) 329-333.

Coleman, S.B., H.B. Clay, D.G. McCurdy, H.L. Norris III. 1991c. Understanding gas-well load-up behavior. Journal of Petroleum Technology 43(3) 334-338.

Cortazar, G., E.S. Schwartz, J. Casassus. 2001. Optimal exploration investments under price and geologicaltechnical uncertainty: A real options model. R\&D Management 31(2) 181-189.

Dixit, A.K., R.S. Pindyck. 1994. Investment under Uncertainty. Princeton University Press, Princeton, New Jersey.

Dugan, T. 2006. Personal communication.

Durrer, E.J., G.E. Slater. 1977. Optimization of petroleum and natural gas production - A survey. Management Science 24(1) 35-43.

Fisher, M. 2007. Strengthening the empirical base of Operations Management. Manufacturing ES Service Operations Management 9(4) 368-382.

Garb, F.A., T.A. Larson. 1989. Valuation of oil and gas reserves. H.B. Bradley, ed., Petroleum Engineering Handbook, chap. 41. Society of Petroleum Engineers, Richardson, Texas.

Grayson, C.J. Jr. 1960. Decisions under Uncertainty: Drilling Decisions by Oil and Gas Operators. Harvard Business School, Boston, Massachusetts.

Hahn, W.J., J.S. Dyer. 2008. Discrete time modeling of mean-reverting stochastic processes for real option valuation. European Journal of Operational Research 184(2) 534-548.

Jaillet, P., E.I. Ronn, S. Tompaidis. 2004. Valuation of commodity-based swing options. Management Science 50(7) 909-921.

Kamrad, B., R. Ernst. 2001. An economic model for evaluating mining and manufacturing ventures with output yield uncertainty. Operations Research 49(5) 690-699.

Kamrad, B., S. Lele. 1998. Production, operating risk and market uncertainty: A valuation perspective on controlled policies. IIE Transactions 30(5) 455-468. 
Lai, G., M.X. Wang, S. Kekre, A. Scheller-Wolf, N. Secomandi. 2009. Valuation of the real option to store liquefied natrual gas at a regasification terminal. Working paper 2006-E99, Tepper School of Business, Carnegie Mellon University.

Luenberger, D.G. 1998. Investment Science. Oxford University Press, New York, New York.

Lumley, R.R., M. Zervos. 2001. A model for investments in the natural resource industry with switching cost. Mathematics of Operations Research 26(4) 637-653.

Lund, M.W. 2000. Valuing flexibility in offshore petroleum projects. Annals of Operations Research 99(1) $325-349$.

Moel, A., P. Tufano. 2002. When are real options exercised? An empirical study of mine closings. The Review of Financial Studies 15(1) 35-64.

Olson, T.E., G. Stensland. 1988. Optimal shutdown decisions in resource extraction. Economics Letters 26(3) 215-218.

Routledge, B., D. Seppi, C. Spatt. 2000. Equilibrium forward curves for commodities. Journal of Finance 55(3) 1297-1338.

Schwartz, E., J.E. Smith. 2000. Short-term variations and long-term dynamics in commodity prices. Management Science 46(7) 893-911.

Schwartz, E.S. 1997. The stochastic behavior of commodity prices: Implications for valuation and hedging. Journal of Finance 52(3) 923-973.

Sciullo, F.C. 2006. Personal communication.

Seppi, D.J. 2003. Risk-neutral stochastic processes for commodity derivative pricing: An introduction and survey. E.I. Ronn, ed., Real Options and Energy Management: Using Options Methodology to Enhance Capital Budgeting Decisions, chap. 1. Risk Books.

Slade, M.E. 2001. Valuing managerial flexibility: An application of real-option theory to mining investments. Journal of Environmental Economics and Management 41(2) 193-233.

Smith, J.E. 2005. Alternative approaches for solving real-options problems. Decision Analysis 2(2) 89-102.

Smith, J.E., K.F. McCardle. 1998. Valuing oil properties: Integrating option pricing and decision analysis approaches. Operations Research 46(2) 198-217. 
Smith, J.E., K.F. McCardle. 1999. Options in the real world: Lessons learned in evaluating oil and gas investments. Operations Research 47(1) 1-15.

Smith, J.E., R.F. Nau. 1995. Valuing risky projects: Option pricing theory and decision analysis. Management Science 41(5) 795-816.

Tarek, A.H. 2006. Reservoir Engineering Hanbook. Gulf Professional Publishing.

Trigeorgis, L. 1999. Real Options: Managerial Flexibility and Strategy in Resource Allocation. MIT Press, Cambridge, Massachusetts. 